JOURNAL OF THEORETICAL

AND APPLIED MECHANICS

60, 1, pp. 103-111, Warsaw 2022

https://doi.org/10.15632/jtam-pl/144312

\title{
FILLET EFFECT ON THE BENDING CRASHWORTHINESS OF THIN-WALLED SQUARE TUBES
}

\author{
Zhongyou Xie \\ School of Architectural Engineering, Tongling University, Tongling, Anhui, China \\ Zhixian Zhao, Xiuneng Zhou, Cheng Li \\ School of Rail Transportation, Soochow University, Suzhou, Jiangsu, China \\ Corresponding author: Cheng Li, licheng@suda.edu.cn
}

\begin{abstract}
Filleting four corners of square tubes is suggested to reduce the peak force and improve energy absorbing performance. Three-point bending tests are conducted to investigate fillet radius effects employing an ABAQUS explicit code. Three cases characterized by the ratio of width to thickness are considered. Fillet greatly reduces the maximum forces compared with square cross-sections, and the normalized maximum forces decrease with increasing wall thickness when the fillet radius is larger. Additionally, the fillet dramatically improves $S E A$ (Specific Energy Absorption). The normalized CFE (Crash Load Efficiency) significantly exceeds that of the square ones, and the normalized $C L E \mathrm{~s}$ are almost identical with the increasing fillet radius.
\end{abstract}

Keywords: thin-walled square tubes, bending crashworthiness, fillet effect, three-point bending, finite element analysis

\section{Introduction}

Thin-walled tubes and thin-walled tube based low-dimensional structures and systems are capable of absorbing substantial energy at the cost of light weight, and they are widely used in many engineering fields. Energy absorption performance and structural vibration energy harvesting of thin-walled tubes under axial or oblique compression have been extensively investigated. Thin-walled square tubes subjected to a transverse load are commonly seen, thus a large initial peak reactional force arises, which results in severe damage to protected objects. Wierzbicki and Abramowicz (1983) developed a self-consistent model to illustrate the crushing response of a kind of thin-walled structures. Abramowicz and Jones (1986) carried out a series of axial crushing tests on static or dynamical behavior of circular and square tubes, and developed approximate analytical solutions for progressive buckling. Lim and Liew (1994) derived a Ritz formulation for flexural vibration of shallow cylindrical shells containing thin-walled tubes. Langseth and Hopperstad (1996) conducted static and dynamic tests to study axial crushing behavior of thin-walled square tubes, and deformation modes under two loading conditions were compared. Han and Park (1999) analyzed oblique crushing behavior of thin-walled square tubes, and the relationship of mean crush loads to load angles was revealed. Kim and Wierzbicki (2001) also investigated the oblique crushing response of thin-walled tubes, and established failure loci of the sectional force and moment. Zarei and Kröger (2007) optimized square and circular tubes for maximum energy absorption by means of a multi-design optimization technique. Ding et al. (2014) studied vibrational behavior of a vehicle-pavement coupled system with a nonlinear foundation involving a large number of thin-walled tubes. Recently, some new research work in this field was investigated by Yang et al. (2018) and $\mathrm{Hu}$ et al. (2021). 
In thin-walled tubes, transverse loads leading to bending deformation are frequent in practical applications. The study of the bending collapse behavior is also focused on the previous research. Wierzbicki et al. (1994) derived moment-rotation solutions for square tubes in the bending collapse stage using a simple kinematic model. Kim and Reid (2001) developed an analytical solution for the moment-rotation relationship in terms of a new collapse mechanism. Huang and $\mathrm{Lu}(2005)$ presented an empirical design formula for the bending moment of thin-walled square tubes based on dimensional analysis theory and numerical simulation results. Chen and Masuda (2016) calculated post-buckling strength of rectangular tubes with a large aspect ratio of web to flange using an effective width concept. Zhang et al. (2016) investigated static and dynamic behavior of a type of folded tubes under three-point bending experimentally and numerically. They also studied the bending collapse response of thin-walled square tubes with variational thickness in the cross-section, and optimized thin-walled beams using a response surface method (Zhang et al., 2016). Huang and Zhang (2018) proposed an analytical method to predict the bending collapse response of thin-walled rectangular beams under three-point bending based on Kecman's model.

Previous studies (Wierzbicki et al., 1994; Kim and Reid, 2001) revealed that thin-walled square tubes generally generate a large initial peak reactional load with a great drop when subjected to transverse loads. The large initial peak load perhaps results in severe damage to the protected objects by an energy absorber. In the paper, filleting thin-walled square cross-sections is suggested to lower the initial peak load and improve the energy absorbing performance. Three-point bending tests have been conducted to investigate the effect of fillet radius on the bending crashworthiness employing an ABAQUS explicit code. Three cases characterized by the ratio of width to thickness of square tubes are considered.

\section{Structural and test design}

Thin-walled square tubes with width $b$ and wall thickness $t$ are considered here. It is suggested that the four corners of the square cross-section are filleted into arcs with the same radius $r$, see Fig. 1b. When the radius is zero, the cross-section is square, while the radius increases up to $0.5 b$, the cross-section becomes a typical circle, as shown in Figs. 1a and 1c, respectively.

(a)

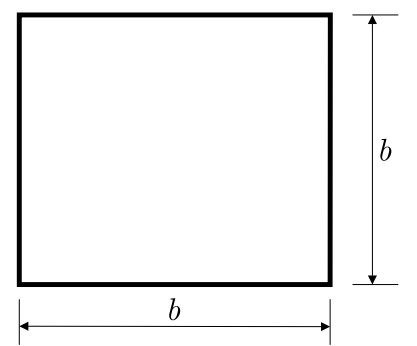

(b) $r$

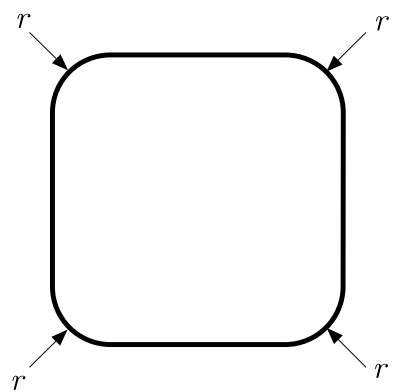

(c)

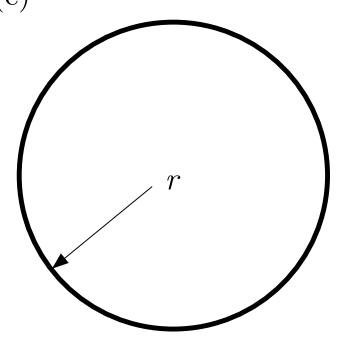

Fig. 1. Variation of the square cross-section with fillet radius: (a) $r=0$, square; (b) $0<r<0.5 b$;

(c) $r=0.5 b$, circular

To investigate the fillet effect on the bending crashworthiness of the thin-walled tubes, three-bending tests are proposed, see Fig. 2. The thin-walled tubes with width $b$ and total length $L$ are supported by two cylindrical supports with spacing $s$, and loaded by a cylindrical punch at the mid-span. The two supports and the punch have the same radius $R$.

In the present analysis, the width of the square $b$ is always kept constant $b=0.1 \mathrm{~m}$, while other geometric dimensions of the tubes are detailed in Table 1 based on the width $b$. The total length $L$ and the supporting span $s$ is $10 b=1 \mathrm{~m}, 8 b=0.8 \mathrm{~m}$, respectively. The radius of the 


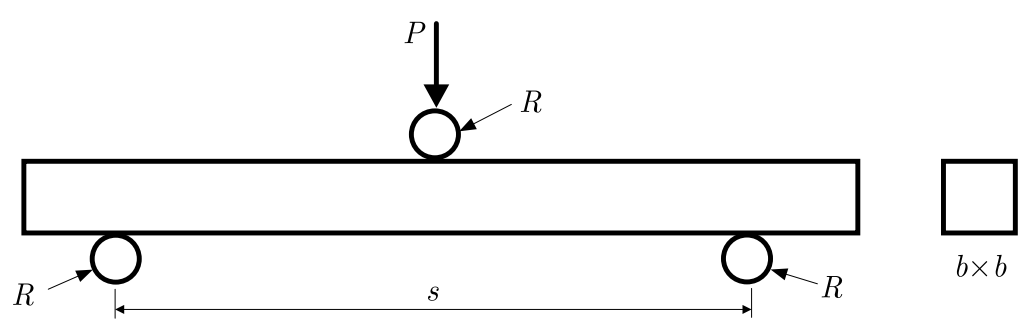

Fig. 2. Schematic of the three-point bending test on thin-walled square tubes

punch and the supports is $0.2 b=20 \mathrm{~mm}$. Thin-walled square tubes are generally characterized by the ratio $b / t$. Three cases of wall thickness denoted by $b / t=100,50,33$, corresponding to the wall thickness $t=1,2,3 \mathrm{~mm}$, respectively, are analyzed in the paper.

Table 1. Geometric dimensions of the numerical tests

\begin{tabular}{|c|c|c|c|c|}
\hline Cases & $\begin{array}{c}\text { Wall thickness } \\
t\end{array}$ & $\begin{array}{c}\text { Total length } \\
L\end{array}$ & $\begin{array}{c}\text { Supporting span } \\
s\end{array}$ & $\begin{array}{c}\text { Cylinder radius } \\
R\end{array}$ \\
\hline \hline \multicolumn{1}{|c|}{$b / t=100$} & $0.01 b=1 \mathrm{~mm}$ & \multirow{2}{*}{$10 b=1 \mathrm{~m}$} & \multirow{2}{*}{$8 b=0.8 \mathrm{~m}$} & \multirow{2}{*}{$0.2 b=20 \mathrm{~mm}$} \\
\cline { 1 - 2 }$b / t=50$ & $0.02 b=2 \mathrm{~mm}$ & & \\
\cline { 1 - 2 }$b / t=33$ & $0.03 b=3 \mathrm{~mm}$ & & & \\
\hline
\end{tabular}

\section{Finite element models}

The three-bending tests are conducted using the ABAQUS explicit code. Considering symmetry of the tests, a quarter models are established by applying symmetric boundary conditions in the $x$ and $z$ directions to shorten the computing time, as illustrated in Fig. 3.

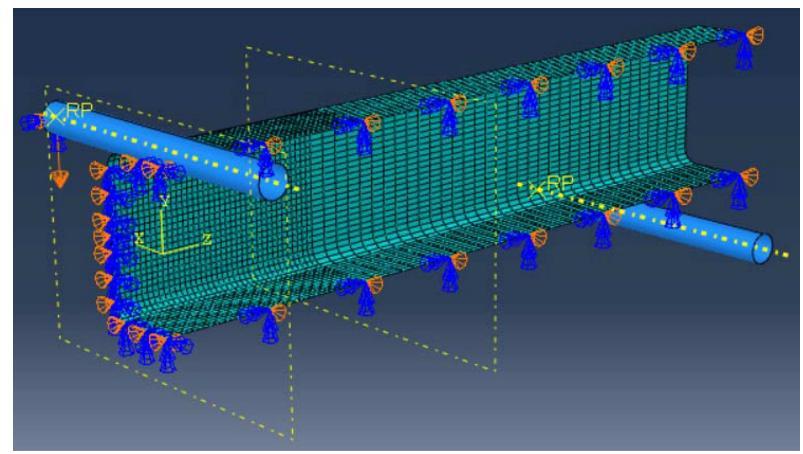

Fig. 3. A quarter model of the bending tests by applying symmetric boundary conditions

In the numerical simulation, the thin-walled tubes are modeled by making use deformable 4-node shell (S4R) elements, with the number 7600 or so. The cylindrical punch and the two supports are simulated by the Analytical Rigid model without any deformation. The tubular wall material is assumed to be a perfectly elastic-plastic metal with density $7.8 \cdot 10^{3} \mathrm{~kg} / \mathrm{m}^{3}$, elastic modulus $200 \mathrm{GPa}$, Poisson's ratio 0.3, and yield stress $600 \mathrm{MPa}$. The Dynamic Explicit procedure is employed to model quasi-static loading by applying a low loading speed. The total time period is $1 \mathrm{~s}$, and the punch moves vertically downwards up to a displacement of $0.1 \mathrm{~m}$ leading to a low constant velocity of $0.1 \mathrm{~m} / \mathrm{s}$. The velocity $0.1 \mathrm{~m} / \mathrm{s}$ is verified to model the quasistatic behavior through adjusting. The support is fully fixed without any displacement. The whole contact behavior is described using general contact with frictionless tangential behavior 
and hard normal behavior. Considering that the deformation mainly occurs in short length of tubes across the mid-span, this zone is meshed with fine size, while other zones are meshed with coarser size, see Fig. 3. A half of tubular length $0.15 \mathrm{~m}$ is meshed with size about 2-3 times of wall thickness $2 t-3 t$ determined through convergence analysis, while the mesh size of other zones is about $5 t$.

\section{Punch force characteristic and stress-stain distribution}

Figure 4 gives the punch force-displacement curves for the three cases $b / t=100,50,33$. For the case $b / t=100$, it can be seen that the square tube has a high elastic peak force followed by a great drop, as depicted in Fig. 4a. The response history before the high peak force corresponds

(a)

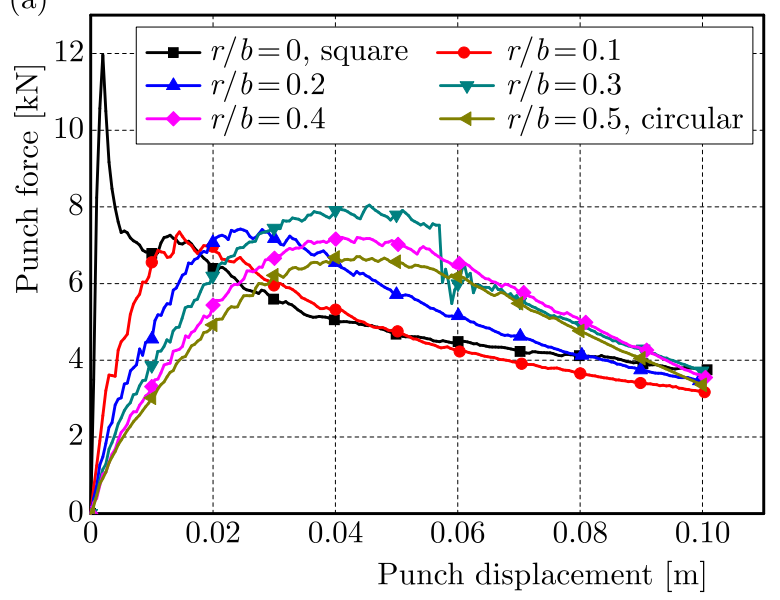

(b)

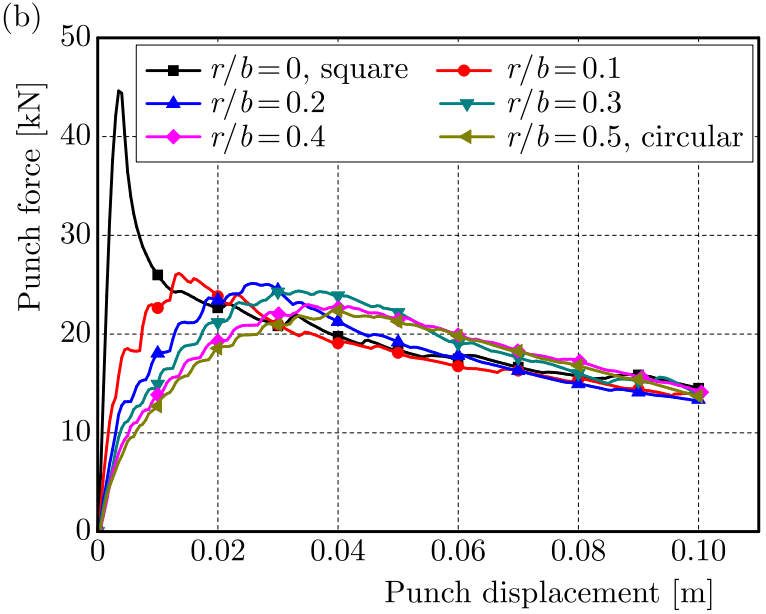

(c)

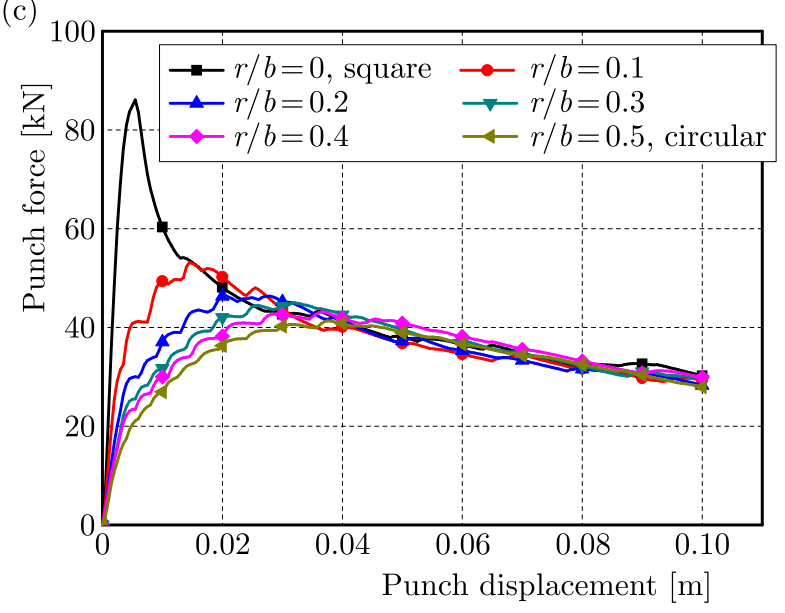

Fig. 4. The punch force-displacement curves for the three cases: (a) $b / t=100 ;$ (b) $b / t=50$; (c) $b / t=33$

to the elastic bending deformation, where the moment of inertia of the cross-section $I_{z c}$ is the key characteristic quantity. The paper focuses on the energy dissipation ability of thin-walled structures, so the elastic indices such as $I_{z c}, E_{t} / I_{z c}$ are not analyzed herein. The numerical results indicate that the deformation of square tubes mainly concentrates in a very short range in the longitudinal direction, leading to a great decrease of the cross-sectional height and bending resistance. When the corners are filleted, no typical elastic response is observed, and the maximum force significantly decreases. The maximum force of the fillet case $r / b=0.3$ is the largest one among all of the filleted tubes. The curve of the case $r / b=0.1$ approaches close to that of the square tube after the maximum force. In addition, for a larger fillet radius $r / b>0.1$, 
the curves are prominently higher than that of the square tube within some range of loading process. The displacement corresponding to the maximum force increases with the increase of the fillet radius when $r / b \leqslant 0.3$. For the two cases $b / t=50,33$, the maximum force of the fillet case $r / b=0.1$ is the largest one among all of the filleted tubes, see Figs. $4 \mathrm{~b}$ and $4 \mathrm{c}$. For the case $b / t=33$, it is noted that all of the curves of the five filleted tubes are very close to that of the square tube in the later loading stage.

Figures $5 \mathrm{a}$ and $5 \mathrm{~b}$ gives the von Mises stress and equivalent plastic strain distribution in the tubular wall for the case $b / t=100$ with $r / b=0.3$ when the punch displacement arrives at the maximum value $0.1 \mathrm{~m}$. It can be found that the deformation mainly occurs in a short region across the mid-span, corresponding to very large stress. A dramatic Brazier effect occurs, and the cross-sectional height at the mid-span cross-section as well as the punch forces largely decrease. The Brazier effect is apt to take place in thin-walled tubular beams due to lack of an internal bearing in the upper compressed zone. On the other hand, it is noted that a lateral inward deformation appears at a certain distance from the mid-span, which may help dissipate more plastic stain energy. Furthermore, the equivalent plastic strain at the mid-span and lateral inward regions prominently surpass the one in other regions, according to Fig. 5b. It is noticed that not only the cross-section changes with $r / b$ in the present model, but also the moment of inertia varies due to the change of cross-section. However, the change of the moment of inertia can be neglected because the present study is only concerned with the large flexible deformation of thin-walled tubes in order to explore their plastic strain energy absorbing capacity. There is little effect of the moment of inertia and elastic deformation on the plastic strain energy absorption.
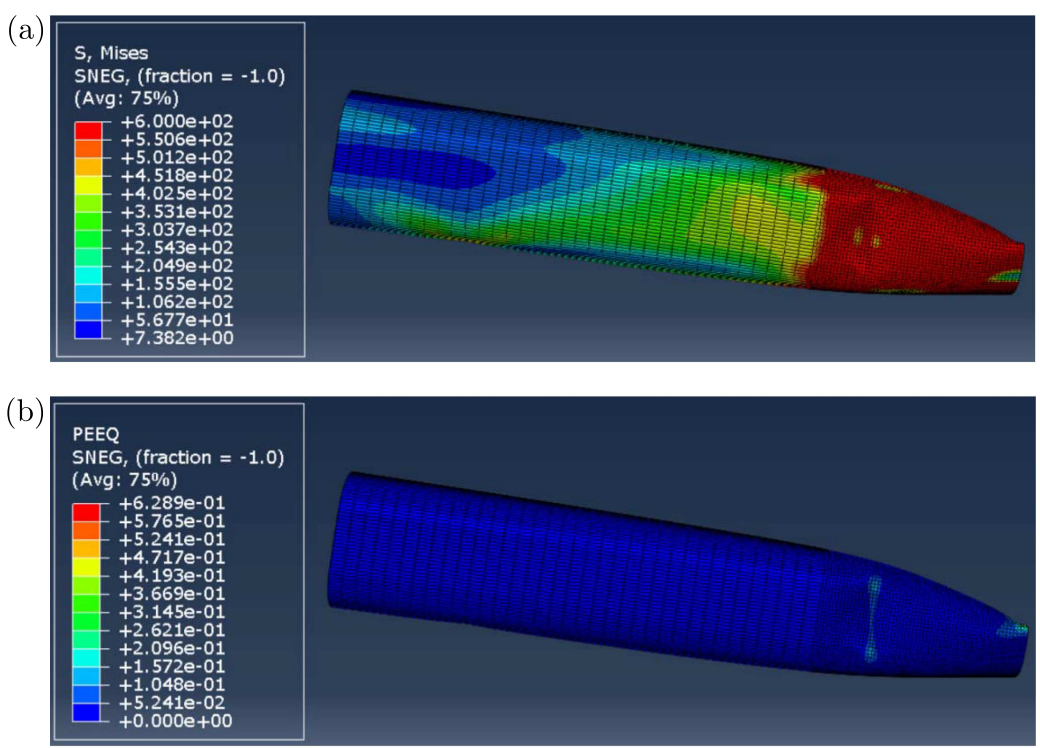

Fig. 5. The stress and strain distribution for the case $b / t=100$ with $r / b=0.3$ under the maximum punch displacement $0.1 \mathrm{~m}$ : (a) von Mises stress; (b) equivalent plastic strain

\section{Bending crashworthiness}

Based on the principle of energy conservation, the work done by the punch will be transferred into the structural internal energy $E_{t}$, i.e.

$$
E_{t}=\int_{0}^{\delta_{\max }} P d \delta
$$


where $P, \delta$ are the punch force and displacement, respectively. The maximum displacement $\delta_{\max }$ takes the value $0.1 \mathrm{~m}$ here. The mean force $P_{m}$ is defined by the ratio of the total internal energy $E_{t}$ to the maximum displacement $\delta_{\max }$

$$
P_{m}=\frac{E_{t}}{\delta_{\max }}
$$

It is desired that more collision energy can be absorbed at the cost of a slight increase in the structural mass. The specific energy absorption $S E A$ was proposed to evaluate the structural energy absorbing performance of the unit mass as follows (Kim, 2002)

$$
S E A=\frac{E_{t}}{m}
$$

Additionally, a too high peak force would result in severe damage to the protected objects for the given energy absorption. The crash load efficiency $C L E$ was put forward to evaluate the safeguarding performance, defined by the ratio of the mean force $P_{m}$ to the maximum one $P_{\max }$ (Zarei and Kröger, 2007)

$$
C L E=\frac{P_{m}}{P_{\max }}
$$

Table 2 provides the calculated results of the above four indices for the three cases based on the numerical results. Considering that the tubes with the same material have the same total length, the structural mass is controlled by the total length of cross-sectional segments $l_{c}$ for given wall thickness. Thus, the structural mass can be substituted by the segmental length $l_{c}$ for simplicity of the calculation of $S E A$, i.e. $S E A=E_{t} / l_{c}$.

Table 2. The calculated results of the mechanical indices for the three cases

\begin{tabular}{|c|c|c|c|c|c|c|c|}
\hline Case & $r / b$ & $\begin{array}{c}P_{\max } \\
{[\mathrm{N}]}\end{array}$ & $\begin{array}{c}E_{t} \\
{[\mathrm{~J}]}\end{array}$ & $\begin{array}{c}P_{m} \\
{[\mathrm{~N}]}\end{array}$ & $\begin{array}{c}I_{c} \\
{[\mathrm{~m}]}\end{array}$ & $\begin{array}{c}S E A \\
{[\mathrm{~J} / \mathrm{m}]}\end{array}$ & $\begin{array}{c}C L E \\
{[-]}\end{array}$ \\
\hline \hline \multirow{5}{*}{$b / t=100$} & 0 & 11962 & 520 & 5198 & 0.4 & 1300 & 0.435 \\
\cline { 2 - 8 } & 0.1 & 7349 & 474 & 4742 & 0.383 & 1238 & 0.645 \\
\cline { 2 - 8 } & 0.2 & 7421 & 513 & 5129 & 0.366 & 1402 & 0.691 \\
\cline { 2 - 8 } & 0.3 & 8049 & 567 & 5667 & 0.349 & 1625 & 0.704 \\
\cline { 2 - 8 } & 0.4 & 7211 & 536 & 5362 & 0.331 & 1619 & 0.744 \\
\cline { 2 - 8 } & 0.5 & 6703 & 499 & 4985 & 0.314 & 1589 & 0.744 \\
\hline \multirow{5}{*}{$b / t=50$} & 0 & 44618 & 1982 & 19824 & 0.4 & 4955 & 0.444 \\
\cline { 2 - 8 } & 0.1 & 26158 & 1806 & 18059 & 0.383 & 4715 & 0.690 \\
\cline { 2 - 8 } & 0.2 & 25141 & 1791 & 17905 & 0.366 & 4893 & 0.712 \\
\cline { 2 - 8 } & 0.3 & 24366 & 1849 & 18491 & 0.349 & 5298 & 0.759 \\
\cline { 2 - 8 } & 0.4 & 22984 & 1809 & 18092 & 0.331 & 5465 & 0.787 \\
\cline { 2 - 8 } & 0.5 & 22441 & 1748 & 17484 & 0.314 & 5567 & 0.779 \\
\hline \multirow{5}{*}{$b / t=33$} & 0 & 86108 & 4133 & 41329 & 0.4 & 10333 & 0.480 \\
\cline { 2 - 8 } & 0.1 & 53176 & 3751 & 37510 & 0.383 & 9794 & 0.705 \\
\cline { 2 - 8 } & 0.2 & 46446 & 3593 & 35935 & 0.366 & 9817 & 0.774 \\
\cline { 2 - 8 } & 0.3 & 45060 & 3567 & 35670 & 0.349 & 10220 & 0.792 \\
\cline { 2 - 7 } & 0.4 & 43156 & 3541 & 35419 & 0.331 & 10698 & 0.821 \\
\cline { 2 - 7 } & 0.5 & 41353 & 3371 & 33712 & 0.314 & 10736 & 0.815 \\
\hline
\end{tabular}

Figure 6a demonstrates the variation of the normalized maximum forces of the punch with fillet radius. For convenient comparison, all of the maximum forces are divided by that of the square tube with identical wall thickness. The following three indices (normalized mean force, 
normalized $S E A$, normalized $C L E$ ) are defined according to the same way. It can be found that the fillets greatly reduce the maximum force, which makes significant meaning for safeguard of the protected objects. For the three cases, the normalized maximum forces are almost identical at $r / b=0.1$. For the case $b / t=100$, the maximum force at $r / b=0.3$ is the largest one among all of the filleted tubes. The normalized maximum forces of the two cases $b / t=50$ and 33 go very close to each other with an increase of the fillet radius $r / b$.
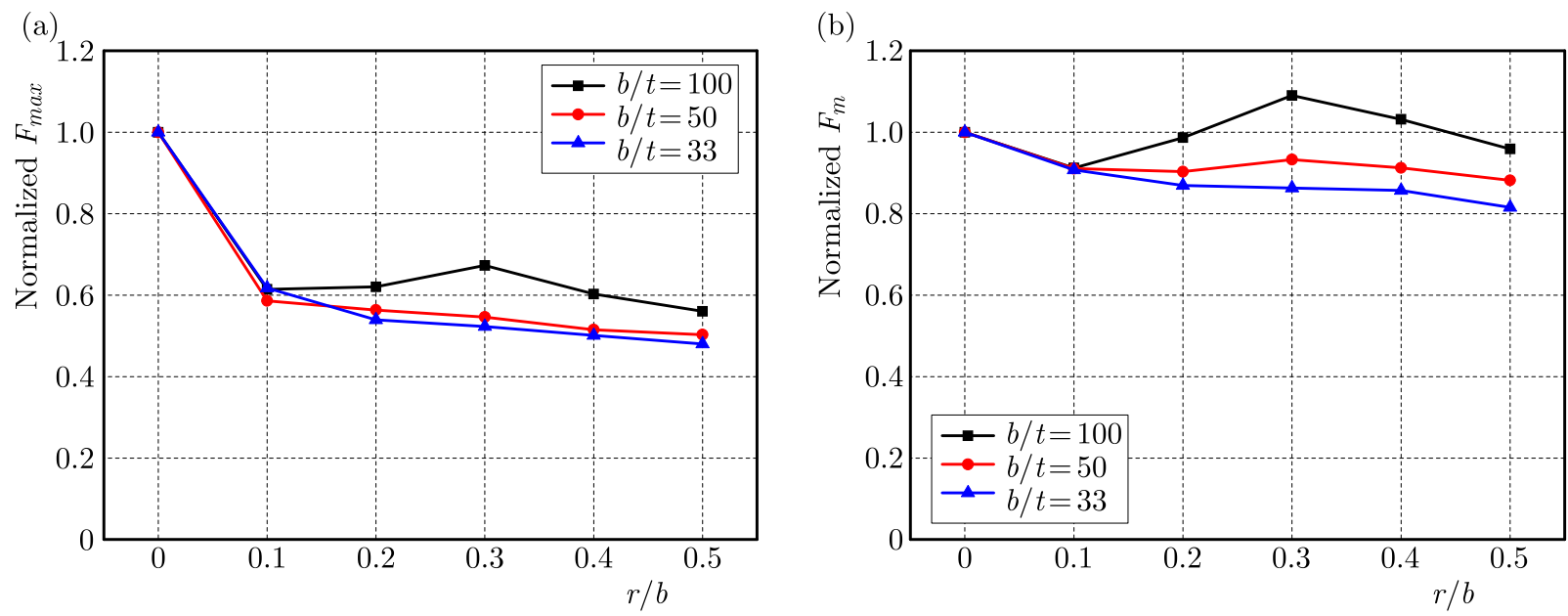

Fig. 6. Variation of the normalized (a) maximum forces and (b) mean forces of the punch with fillet radius

Figure $6 \mathrm{~b}$ shows variation of the normalized mean forces of the punch with fillet radius. The variational characteristic of the normalized mean forces is similar to that of the normalized maximum one. It is different that the normalized mean forces of the case $b / t=33$ are a little smaller than those of the case $b / t=50$ when $r / b>0.1$.

(a)

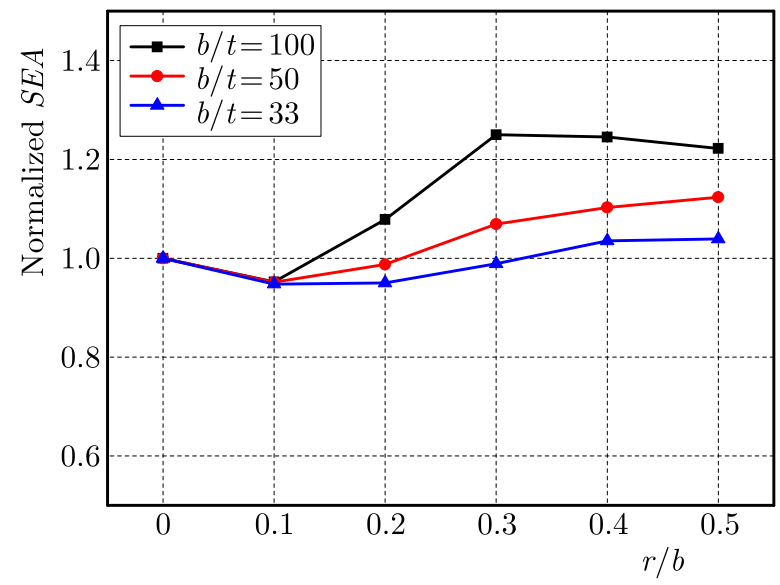

(b)

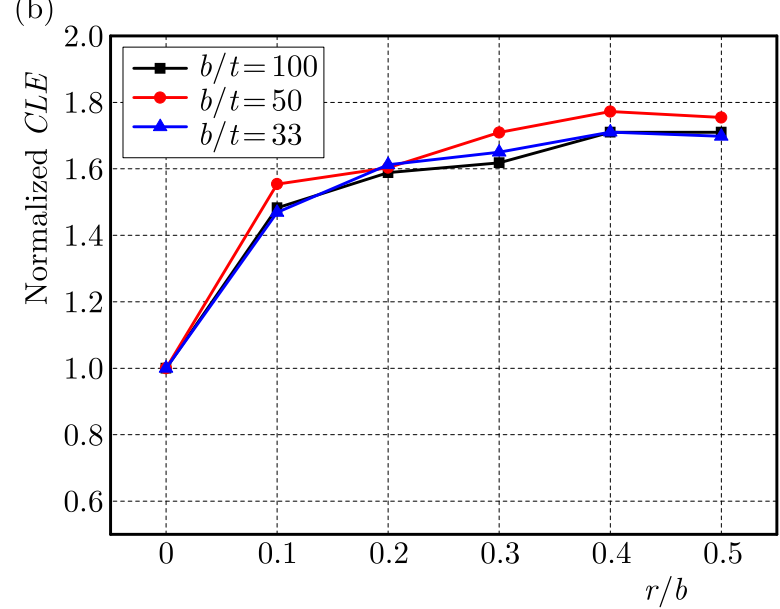

Fig. 7. Variation of the normalized (a) $S E A$ and (b) $C L E$ with fillet radius

Figure 7a depicts the variation of the normalized $S E A$ with fillet radius. It can be seen that the fillet dramatically improves $S E A$ compared with the square tubes, especially when the wall thickness is smaller, i.e. the case $b / t=100$. The normalized $S E A$ of the three cases are almost equal and a little smaller than those of the square tubes at $r / b=0.1$. For the case $b / t=100$, the normalized $S E A$ considerably rises with an increase of the fillet radius when $r / b>0.1$, and reaches the peak value at $r / b=0.3$. It is noticeable that the circular cross-section, i.e. $r / b=0.5$, exhibits an about $22 \%$ larger $S E A$ than that for the square cross-section. For the two cases 
$b / t=50,33$, the normalized $S E A$ keeps a rising tendency when $r / b>0.1$, and gets to the peak values at $r / b=0.5$, i.e. for circular cross-section. It can be concluded that the circular cross-section is superior to the square cross-section with identical $b / t$ in terms of the index $S E A$.

Figure $7 \mathrm{~b}$ displays variation of the normalized $C L E$ with fillet radius. It can be observed that the normalized $C L E$ of the filleted tubes significantly exceeds that of the typical square cross-sections. Additionally, it is interesting that the normalized $C L E$ of the three cases are almost identical with the increase of fillet radius. It is noted that the circular cross-sections display about $70 \%$ larger $C L E$ s than the square cross-sections.

\section{Conclusions}

Thin-walled square tubes are widely used in many engineering fields. When subjected to transverse loads, the tubes would undergo bending deformation. A large initial peak reactional force would arise, which perhaps brings about severe damage to the protected objects. In the paper, filleting the square cross-section is suggested to decrease the maximum reactional force. Three-point bending tests are conducted to investigate the effect of fillet radius on the bending crashworthiness of the filleted tubes by employing an ABAQUS explicit code. All of the thin-walled tubes are supposed to have the same material, width and total length. The bending crashworthiness of three cases of wall thickness characterized by $b / t=100,50,33$ are compared based on the four indices: the maximum force, mean force, $S E A$ and $C L E$.

It is found that the fillet greatly reduces the maximum forces compared with its original square cross-sections, and the normalized maximum forces decrease with an increase of wall thickness when the fillet radius is larger. Additionally, the fillet dramatically improves $S E A$ performance, especially when the wall thickness is smaller. It is noticeable that the circular cross-section is superior to the square cross-section in terms of the index $S E A$. Moreover, the normalized $C L E$ of the filleted tubes significantly exceeds that of the square ones, and the normalized $C L E$ of the three cases are almost identical with an increase of fillet radius. It is noted that the circular cross-sections display about $70 \%$ larger $C L E$ than the square cross-sections.

\section{Acknowledgement}

This work was supported by University Natural Science Research Project of Anhui Province (No. KJ2018A0481), the Natural Science Foundation of China (No. 11972240), and the China Postdoctoral Science Foundation (No. 2020M671574), which are gratefully acknowledged.

\section{References}

1. Abramowicz W., Jones N., 1986, Dynamic progressive buckling of circular and square tubes, International Journal of Impact Engineering, 4, 4, 243-270

2. Chen D.H., Masuda K., 2016, Estimation of collapse load for thin-walled rectangular tubes under bending, Journal of Applied Mechanics, 83, 3, 10-12

3. Ding H., YANG Y., Chen L.Q., YANG S.P., 2014, Vibration of vehicle-pavement coupled system based on a Timoshenko beam on a nonlinear foundation, Journal of Sound and Vibration, 333, 24, 6623-6636

4. HAN D.C., PARK S.H., 1999, Collapse behavior of square thin-walled columns subjected to oblique loads, Thin-Walled Structures, 35, 3, 167-184

5. Hu W., Xu M., Song J., Gao Q., Deng Z., 2021, Coupling dynamic behaviors of flexible stretching hub-beam system, Mechanical Systems and Signal Processing, 151, 107389 
6. HuAng X., Lu G., 2005, Bending hinge characteristic of thin-walled square tubes, International Journal of Crashworthiness, 10, 3, 275-285

7. Huang Z., Zhang X., 2018, Three-point bending collapse of thin-walled rectangular beams, International Journal of Mechanical Sciences, 144, 461-479

8. KIM H.S., 2002, New extruded multi-cell aluminum profile for maximum crash energy absorption and weight efficiency, Thin-Walled Structures, 40, 4, 311-327

9. Kim H.S., WierzBicki T., 2001, Crush behavior of thin-walled prismatic columns under combined bending and compression, Computers and Structures, 79, 15, 1417-1432

10. Kim T.H., REID S.R., 2001, Bending collapse of thin-walled rectangular section columns, Computers and Structures, 79, 20-21, 1897-1911

11. LAngseth M., Hopperstad O.S., 1996, Static and dynamic axial crushing of square thin-walled aluminium extrusions, International Journal of Impact Engineering, 18, 7-8, 949-968

12. Lim C.W., LiEw K.M., 1994, A pb-2 Ritz formulation for flexural vibration of shallow cylindrical shells of rectangular planform, Journal of Sound and Vibration, 173, 3, 343-375

13. WierzBicki T., Abramowicz W., 1983, On the crushing mechanics of thin-walled structures, Journal of Applied Mechanics, 50, 727-734

14. Wierzbicki T., Recke L., Abramowicz W., Gholami T., Huang J., 1994, Stress profiles in thin-walled prismatic columns subjected to crush loading - II Bending, Computers and Structures, $\mathbf{5 1}, 6,625-641$

15. YAng X.D., YAng J.H., Qian Y.J., Zhang W., Melnik R.V.N., 2018, Dynamics of a beam with both axial moving and spinning motion: An example of bi-gyroscopic continua, European Journal of Mechanics-A/Solids, 69, 231-237

16. Zarei H.R., KröGer M., 2007, Crashworthiness optimization of empty and filled aluminum crash boxes, International Journal of Crashworthiness, 12, 3, 255-264

17. Zhang X., Zhang H., Ren W., 2016, Bending collapse of folded tubes, International Journal of Mechanical Sciences, 117, 67-78

18. Zhang X., Zhang H., WAng Z., 2016, Bending collapse of square tubes with variable thickness, International Journal of Mechanical Sciences, 106, 107-116 